\title{
End to End Development Engineering
}

\author{
Abdelgaffar Hamed ${ }^{1}$, Robert M. Colomb ${ }^{2}$ \\ ${ }^{1}$ College of Computer Science and Information Technology, Sudan University of Science and Technology, Khartoum, Sudan; \\ ${ }^{2}$ Faculty of Computer Science and Information Systems, Universiti Technologi Malaysia, Skudai, Malaysia. \\ Email: abdalgafarhamid@sustech.edu,colomb@siswa.utm.my
}

Received February 22 ${ }^{\text {nd }}, 2011$; revised March $10^{\text {th }}, 2011$; accepted March $13^{\text {th }}, 2011$.

\begin{abstract}
Raising software abstraction and re-use levels are key success factors for producing quality software products. Modeldriven architecture (MDA) is an OMG initiative following this trend by mapping a conceptual model of application specified in platform independent model (PIM), to one or more platform specific models (PSM) automatically. Because there is little previous work tackling the development problem from specification through to implementation, this paper proposes End to End Development engineering (E2EDE) method using MDA methodology. E2EDE is intended to fill the mapping gap between PIM and PSM in MDA. The notion of variability is utilized from software product line and used to model design decisions in PSM. PIM is equipped with Nonfunctional requirements which borrowed from Design pattern to inform design decisions; thereby guiding the mapping process. In addition we have developed a strategic PSM for messaging systems can be configured to produce different applications such as the helpdesk system which is used as a case study.
\end{abstract}

Keywords: End to End Engineering, MDA, Metamodeling, Domain Engineering

\section{Introduction}

The complexity of producing large-scale software systems is increasing due to the increased complexity of requirements. Technologies are volatile for many reasons but enhancing the quality of services is among clear reasons justified by software providers. For example, java platform versions and Google chrome browser has adopted new browsers technology. The functionality of browsers is already crafted (i.e. Mozilla) but putting it into a new fashion is because of security, performance, reliability, and etc. On other hand, service-based system (SOA) has emerged as new engineering discipline encourages organizations to integrate their systems in a seamless manner. These highlight questions like 1) How to extend the traditional methods (Code-based) in a longlived architecture to deliver these new businesses? 2) how to provide an effective integration with legacy systems? 3) If a decision is made to change technology (acquiring new quality such as security and performance) is the design easily adaptable? The trend now is proposing model-based engineering approach which means separating concerns where software development is driven by a family of high level languages [1]. To this end abstraction level is raised above 3GLs which increases re-using theme and put software artifact into a situation of core asset. More importantly formalizing of these artifacts (i.e. metamodels) leads to realize the benefit of high degree of automation. This means a machinery of specification (i.e. UML), synchronization and management of these models are essential. Thus, software not a program became like an information system itself. Thereby the crafting of code is becoming a manufacturing process not a personal skill.

Model Driven Architecture (MDA) is a new software development method following that trend. It raises abstraction level and maximizes re-use. Using MDA, we will be able to work with software artifacts as assets which from the software engineering perspective is a major success factor for reliable and fast development. The philosophy of MDA is to do more investment on software artifacts (models) to increase their efficiency, leading to systematic and more powerful mechanisms for software re-use.

MDA is an aspect of the more general discipline of software reuse. The synergistic relationship among MDA and the longer-established areas of Design pattern and software product line engineering (SPL) [2] has been studied as part of the present research $[3,4]$.

The problem of producing a complete solution from specification through to implementation is still a long 
standing research aim, and because of the mapping gap from PIM to PSM, E2EDE has emerged. Most of previous work in MDA has been on infrastructure and components. Therefore the major question in this paper is how to write a program in MDA?

End to End development engineering (E2EDE) is a new trend to software engineering, proposed to answer that question, which uses MDA methodology and exploits some experience from Software Product Line (SPL) and lessons from Design Pattern (e.g. nonfunctional requirements) to automate the development from specification through to implementation. In doing so, we need to investigate the relationships among MDA, SPL, and design pattern and how MDA can fit on them. Therefore the contributions of the paper are the following:

1) We present E2EDE to automate the mapping process as realization to MDA which is intended to produce products without entirely writing code.

2) We discuss the relationship between MDA, SPL, and design pattern and how MDA can fit in the both longer established re-using approaches.

3) We share some lessons learned and challenges of MDA software engineering in practice.

Domain engineering is the key concept utilized from SPL which realizes breeding of a number of products that have similarity and some sort of variability in features. Design pattern in the history of software engineering has concerned with linking the design with nonfunctional requirements. Therefore, Nonfunctional requirements is borrowed as a concept.

The paper is organized as follows: Section 2 describes MDA as a major method used in E2EDE. In Section 3 we explain the problem through example of mappings from QVT specification. The proposed E2EDE methodology is discussed at Section 4. The concepts of E2EDE are validated by case study at Section 5. Sections 6 and 7 describe the relationship among MDA, Design Pattern and SPL. Section 8 draws on the principle of MDA and shows a case of strategic PSM. In Sections 9 and 10 we discuss MDA and E2EDE implementation aspects respectively. In Section 11 the realistic value of our approach with existing platforms is investigated. A conclusion is presented in Section 12.

\section{Model Driven Architecture (MDA)}

MDA is a new development paradigm initiated by the OMG aimed at software development driven by model [1]. In this case, a Platform Independent Model (PIM) is used to specify application behavior or logic by using MOF or a MOF-complaint modeling language [3]. This step represents a problem space in an application-oriented perspective. A Platform Specific Model (PSM) is used to realize a PIM. It represents a solution space from an imple- mentation-oriented point of view. Therefore, a transformation from the problem space to the solution space is required. The automation of this process is the ultimate goal of MDA. Thereby, when we need to change the application, changes will be in only one part (PIM) without affecting implementation technologies (PSM). Conversely, when the platform such as SQL Server is changed retargeting a new platform for example new version (has enhanced feature), we need only to select the appropriate PSM and then regenerate the code not only without modifying PIM but also this time re-using most of the transformation. Productivity becomes higher and cost is reduced due to the increased reuse of models. In addition, maintenance becomes cheaper. It is worthwhile to observe here that MDA is working with models as assets that can be reused once the initial investment is made. MDA depends on a well established code-base.

\subsection{MDA Transformation Process}

The transformation from PIM to PSM is done by a maping function, which is a collection of mapping rules. In this case some or all of the content of the target model is defined. It is expected that when MDA automates this process, development efficiency and portability would significantly increase. In addition, the mapping function can be repeated many times (re-used) for different applications using the same PIM and PSM metamodels. MDA also helps avoid risks of swamping the application with implementation detail which causes model divergence [5].

The steps of designing a system is to create a concepttual model by designers for application requirements and developing another implementation model to map the first into the second. But this might involve many sub activities. However, we can divide MDA into two major processes [1]:

1) Model to Model mappings

The mapping in this stage does not consider any specific characteristics or special cases that apply to technology or platform (called M2M). The result of this phase is still high level model but for code (PSM instances).

2) Bringing in a Particular Platform

The goal of this mapping (sometimes called M2T) is tailoring the conceptual model to specific technology. Different platforms have different features and constraints so step 1 will be refined to conform to features of one of the selected platform. The result in this phase is expected to be context dependant code expressed in a platform concrete syntax. In fact, we intended to use the word bringing to denote applying the principle of MDA in developing standard PSM.

\subsection{Metamodeling}

The conceptual model of the design language such as 
UML data model (i.e. class diagram) is called a metamodel, which has concept like a class. A particular design in a design language is called model instance like student class in the student record system. This model instance can be visualized by using UML model instance (i.e. object diagram) but also MOF has similar model instances metamodel. A metamodel defines a schema for database called a repository. The population of this repository is the model instance. Formation rules of the metamodel are expressed as constraint on the repository [6]. A metamodel represents syntax of a modeling language. If the metamodel tells the designer how to create a model instance, it is said to be concrete syntax [6]. If it does not, it is said to be abstract syntax. Therefore, sometimes rendering conventions augmented with abstract syntax to generate concert syntax like MOF instance specification [7].

Model-based design that relies on a repository (tables or data structure) for storing a complex object (design), is the key art behind the MDA approach. For example QVT mappings are specified as patterns on schemas, or metamodel [8]. In this way, information contained in the models is separated from the algorithms defining tool behavior, instead of being hard-coded into a tool. The algorithmic part of tool communicates with models via an abstract program interface (API), which affords the facility to create, modify and access the information in models [9]. Further, MDA tools can transform model instances into various forms. For example, the mappings from PIM to PSM takes PIM metamodel instances from the instance repository and turns it into corresponding instances updating the target repository, moving from problem space to solution space.

This mapping activity is done using standard language independent of the source and target. The metamodel of the mapping from end to another end can also be re-used as an asset.

\subsection{Query, Views, and Transformations (QVT)}

Two kinds of transformation are recognized in MDA community:

- Horizontal, that does not change the abstraction level, for example from PIM to PIM which is used when a model is enhanced, filtered or specialized (mapping from analysis to design),

- Vertical, that changes the abstraction level, for example projection to the execution infrastructure. Four types of these transformations are categorized in [10].

There are tools to specify such mappings, such as query-view-transform QVT [8]. QVT is an OMG standard which helps us to specify rules for the transformation function. QVT uses the concept of predicate (expression evaluated to true or false) and pattern (set of expressions) in much similar way as prolog programming language.

The intended scenario of writing a program using MDA will be demonstrated first by an example then below in our case study where the mapping task is the major activity. Generally the application development is a process involves many transformations so vertical and horizontal or combination of them might be used.

\section{MDA Example and Mapping Problem}

We will use the QVT specification [8] example of object to relational mappings in order to understand where the problem is in this context. For sake of simplicity we focus on part of the mappings between PIM (object model) and PSM (relational model). This example shows the mappings take place between simplified UML2 metamodel in Figure 1, the PIM, and the PSM in Figure 2.

The mapping between conceptual models and relational schema is well established in the database. The general idea is that classes map to tables, packages map to schemas, attributes to columns, and associations to foreign keys. We will discuss part of this mapping informally then we will show simple QVT rules for that.

In Figures $\mathbf{3}$ and $\mathbf{4}$ examples we have a relation (use to specify rules of mapping source to target) named PackageToSchema, and ClassToTable. Both have two domains that will match elements in uml (our PIM) and rdbms (our PSM).The relation ClassToTable specifies the map of a class which has attribute name with value equal the variable “cn”. All classes instances in uml repository will populate this variable one by one. For example if the model instance of our PIM is student record system, these will be person, lecturer, student, etc (M1). If the precondition is satisfied by this way the enforce clause is very similar to a checkonly clause. If there is an instance in the rdbms repository satisfying the pattern expression, then the enforce clause behaves as a predicate, with the value true. If no such instance exists, then the QVT engine will create one.

The mapping takes structural patterns in the M1 PIM model (problem domain) instance into in some cases quite different structural patterns in the M1 PSM model (implementation domain). The patterns are described in the M2 metamodel. Thereby that mapping is grasped as a part of the process of specifying and implementing the system. This process in most traditional software engineering methods is done manually. The ultimate goal of mapping is having a way to be able relate $M 0$ instances of the PIM to M0 instances of the PSM. But the standarddocument of MDA [1] does not specify how to do that.

MOF specification has described how to create instances from MOF-based metamodel. When we talk about MOF we mean M2 level in the OMG hierarchy. Both 


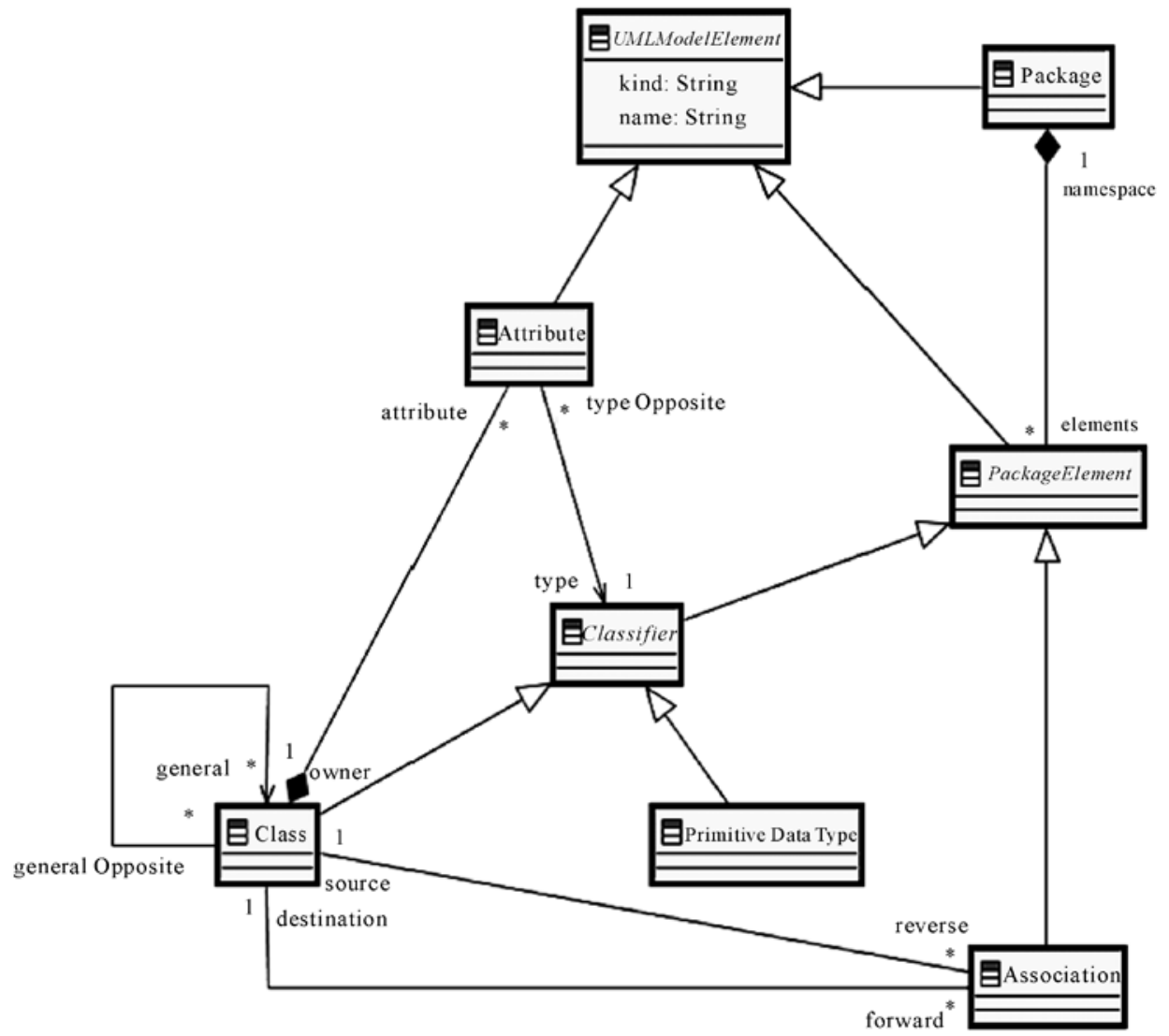

Figure 1. Simplified UML2 metamodel from QVT [8].

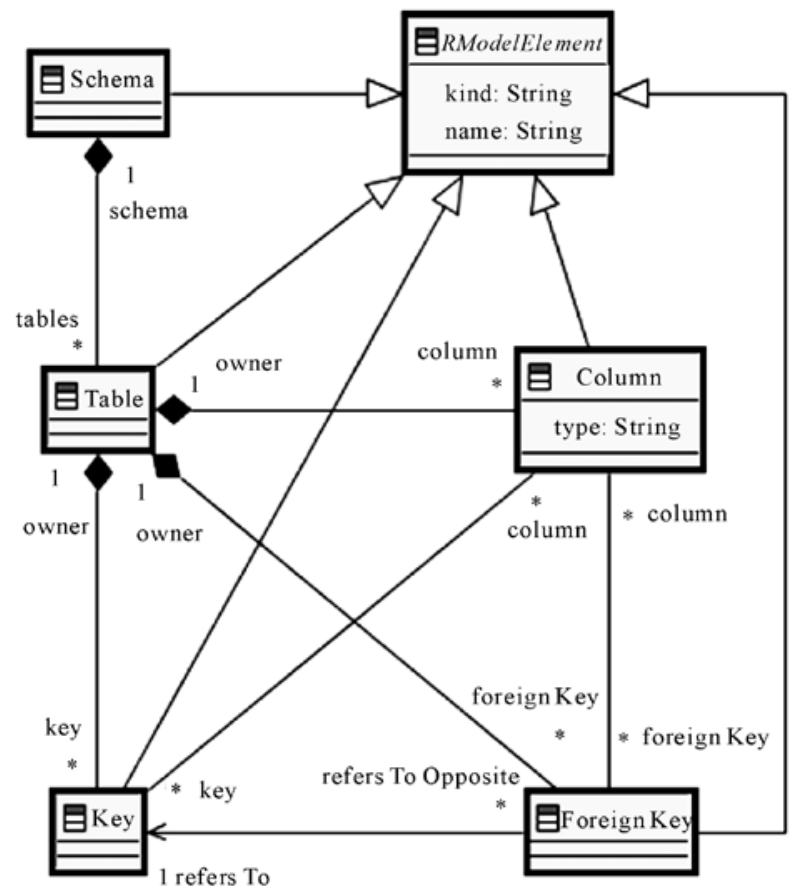

Figure 2. Simplified relational database model from QVT specification [8]. create and destroy methods for objects and links are specified as MOF standard operations for creating and deleting dynamic objects [7]. So the objects created and destroyed by these methods are of kind M1 objects for M2 metaclasses. By this way an instance model will be created for M2 metamodel elements similar like having student, lecturer, and course, etc., instances. UML standard also specifies methods for creating instance model for M1 objects which are M0.Hence since like the instance model of record system is UML instance model then it should be able to create m0 objects using UML model instance inherited capabilities: create and destroy of objects and links.

The intended scenario for mapping is that an application using application terms have a facility to create objects which is PIM instance model. On other hand the implementation model that is PSM creates objects corresponding to that objects using PSM concepts and terms which represents a design vocabulary. To this end still in the MDA development (i.e. using MDA to solve problems) the question is how to do this process which requires finding concrete technical mapping methods.

A general method to approach this problem is needed whereby one could develop application without entirely 


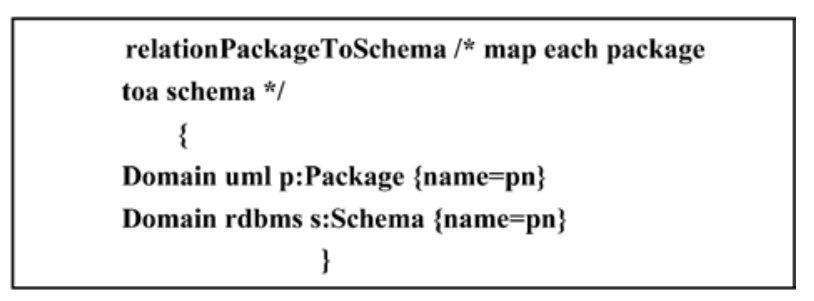

Figure 3. Mapping package to schema QVT rule.

relationClassToTable $/{ }^{*}$ map each persistent class
to a table */
\{
Checkonly domain umlc:Class \{
name $=$ cn $\}$
name $=$ cn $\}$
when $\{$ PackageToSchema(p, s); $\}$
where $\{$ AttributeToColumn(t, c); $\}\}$

Figure 4. Mapping class to table QVT rule.

writing code. Here the task for developer/modeler is a function of specification where application requirements, implementation and mapping metamodels are presented using design languages such as UML/MOF and mapping language such as QVT. In this case mapping and synchronization among models are performed by toolset.

\section{Proposed E2EDE Design \& Implementation}

This section demonstrates key points of E2EDE and explores most important aspects that should be addressed.

\subsection{Introduction}

End to End development engineering (E2EDE) is a novel paradigm intended to automate software development from the specification end (i.e. object model) to the implementation end (i.e. relational model) using the MDA approach. The central issue is filling the mapping gap between PIM and PSM in MDA.

Theoretically E2EDE is inspired from an investigation of the synergistic relationship among MDA, SPL, and design patterns as we will see in Sections 6 and 7.The rationale behind establishing this relationship was from literature SPL and design patterns have long history of re-use software development. So they are longer established reuse methods. MDA is a more recent stream of re-use. E2EDE engineering is going to exploit this relationship to achieve its mapping goal. The key concept in SPL is variability which gives customization or configuration options. Variant feature is a place in the software artifact can be populated by at least one variant at a time from a set of variant. For example, if color is variant feature then Red is one variant. We conceive design decisions as variation points and the design choices as variants populating these points. This comes from the observation that PSM as a design artifact has different structures most properly lead to different architectural qualities. Therefore, to model design decisions we need to represent variability explicitly in the PSM. The study of the design pattern approach highlights the importance of the relationship between design and requirements, specifically nonfunctional requirements, which is proposed to be modeled in the PIM. Still there is a research gap in these areas on how to map nonfunctional requirements with design decisions systematically. The problem has been looked at from one dimension, for example SPL has concerned only with variability without considering NFR such as $[2,3,11$, 12] while design pattern has recognized the impact of NFR dimension without variability in an explicit way [13-15].

However, the benefit here is PSM construction could be automated effectively because of consideration of design quality and management of single PSM. Hence, documenting variability in architecture and modeling nonfunctional requirements explicitly will become major activities during the development process. This section demonstrates key issues arising when we tackle E2EDE. Further, these issues have been applied to a selected case study to evaluate the possibility of the proposed engineering approach.

The ultimate goal of E2EDE is to provide a method of generating a solution from one specific source to a specific target like for example, from object-model to relational model. The advantages of E2EDE are reflected in the modeling support for the concepts in the domain and the ability to do more than general-purpose languages do, in addition to reduction of cost.

\subsection{The Steps of E2EDE Process}

In this section we will see the main steps of E2EDE. It will be detailed step by step and finally summarized as shown in Table 1.

\subsubsection{Modeling Variation Points in PSM (Task A)}

The key concept is to document variability in the PSM, which can be thought of as an abstract data type similar to the logical level in database systems. Usually, a solution is specified firstly at high abstraction level before rendered into a database technology. Variability in this PSM exposes different design decisions from the design space. The design decisions we mean in this context are architectural elements. Since it is possible to create different implementations from a generic specification, variability management concepts and techniques from SPL ex- 
Table 1. The steps of E2EDE process.

\begin{tabular}{ccc}
\hline Tasks & Technique & Specific Solution \\
\hline $\begin{array}{c}\text { A- Modeling Variation Points in PSM } \\
\text { B- Analyzing Variability and categorized based on } \\
\text { PSM /mapping, and functional/non-functional } \\
\text { C- Developing Profiles for variability \&NFRs }\end{array}$ & $\begin{array}{c}\text { variability from SPL } \\
\text { variability concepts + MDA concepts + } \\
\text { design concepts } \\
\text { D-Modeling of Non-functional Requirements in PIM } \\
\text { and classified into package/class level }\end{array}$ & $\begin{array}{c}\text { Guidelines and informal steps help } \\
\text { categorizing different sorts. }\end{array}$ \\
E- Developing model-model mapping rules. & nonfunctional requirements concepts & MOF language \\
F- Packaging mappings variability rules & QVT language & $\begin{array}{c}\text { Profile for PIM + guidelines for } \\
\text { classifying NFRs }\end{array}$ \\
G- Implement the system & opaque rule & QVT rules \\
QVT meta rules
\end{tabular}

periences are utilized to document design decision variants explicitly in the architecture. For example we will see in Section 5 two types of connection: Topic (indirect) and Queue (direct) for a messaging system. The variability difference is that in topic multiple subscribers receive a message while in queue only one subscriber is allowed to receive. This variability can be modeled as two different structures at PSM or formally as variants populating the connectionType variation point.

Since standard UML does not have a variability concept, modeling variability in a PSM we need to use a profile to allow us to specify the variability ontology. A profile is a special domain language used as an extension mechanism to UML model elements while keeping their syntax and semantic intact.Proposed metamodels and profiles in the literature such as [2,12]can allow an architect to identify specific variation points, constraints and dependencies that indicate different relationships between variation points (VP) and variants (V), VP and VP, etc.

Because we are using design model (i.e. class diagram) the proposed profile here is different from these because there is no need for dependency and constraints concepts. They are built-in mechanisms whose semantics is specified with the mapping process and NFRs. Also, there is no need for open and closed concepts which gives the ability to add new variant or variation points because all are closed in this situation (MDA works above 3 GLs).

Therefore, developing a suitable variability MOF-profile is an essential part for the solution presented by E2EDE.In fact there are alternative ways to model variability and nonfunctional requirements concepts using a profile. The method we have chosen will help produce a working system.

The variability ontology needed includes the concepts variant indicated by $\langle<\mathrm{V}\rangle>$ stereotype, variation point indicated by $<<\mathrm{VP}>>$, and an ID tag attribute to identify each VP.

In Figure 5 the UML metaclass class is extended to represent variant and variation point. A tagged value extension mechanism is used to model identifier and type meta-attributes. Tagged values are additional meta-attributes assigned to a stereotype, specified as name-value pairs. They have a name and a type and can be used to attach arbitrary information to model elements.For instance, if we need to model ConnectionType (the two kinds of connections in messaging system) variation point we use the stereotype $<<\mathrm{VP}>>$ and for its variants Queue and Topic we use two $<<\mathrm{V}>>$ at class level. Then the tag for ConnectionType will be VPID =1and default can take the value Direct. The effect tag of variant specifies design decision consequences like resource consumption.

\subsubsection{Variability Analysis (Task $B$ )}

A taxonomy for variability has emerged from our analysis of variability in software architecture artifacts. They could be called Nonfunctional variability, Functional variability and Mapping variability. SPL has been focused mainly on functional variability. An extensive analysis of this can be found in [16]. Although our proposed method includes this sort of variability, it highlights the influence of Nonfunctional variability in the design. Most of the issues discussed in Section 6.3 are of this kind. Mapping variability can be seen in the problem of mapping superclass/subclass structures from object model into relational model. It is not like the others because the variation points are in the transformation, not in the PSM (i.e. the mappings are parameterized). In this case the mapping is from an object model as a source to the relational model as the target. The former specifies objects and relationships between them which may includes superclass-subclass relationship in a class diagram, while the latter specifies relations and their structure. The metamodels and mapping using QVT of these are well described in [8].The target does not include a structure corresponding to superclass-subclass in the source. To solve 


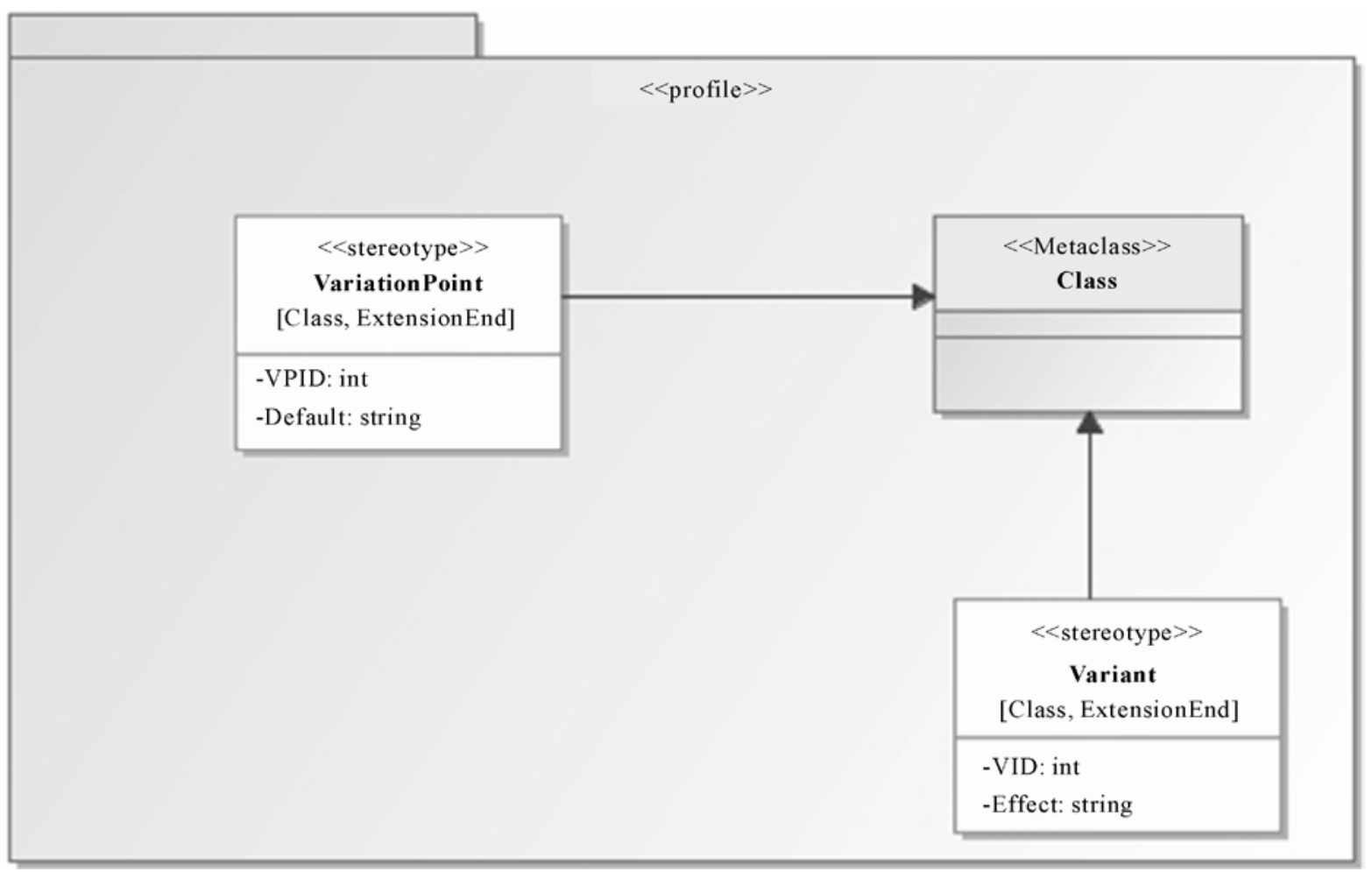

Figure 5. Variability MOF-profiles.

this deficiency four options are suggested for this mapping in the standard database literature [17].

Generally these options can be classified into singlerelation and multiple-relation approaches named SR and $\mathrm{MR}$ respectively. In the SR approach a table for superclass attributes will be created with subclass attributes included as optional while in MR approach table for each subclass will be created. Two implementation techniques are available for both. SR can be implemented by introducing one type attribute indicating the subclass to which each tuple belongs (null values will introduced), or multiple boolean type attributes can be used (allowing overlapping subclasses). MR has as one option with super class attributes duplicated in each subclass table and another option to share a key among superclass and subclass tables.

For example, the option of one table for the superclass with subclass attributes included as optional is a good design in terms of performance for SQL navigation, at a cost of increased table space and increased integrity checking.

\subsubsection{Modeling of Non-Functional Requirements (Task D)}

The E2EDE methodology considers NFRs as first class objects which allow a PIM metamodel to be more informative. The separation of concerns (i.e. PIM-PSM) of MDA effectively supports their representation.

Functional requirements are functions that the devel- oped software must be capable of performing, while nonfunctional requirements (NFRs) inform the design choices as to how functional requirements are going to be realized in software products [16]. There is no one agreed definition because of the extremely diverse nature of NFR. In fact, practices like in design pattern shows a single NFR can have different semantic interpretations (impact on implementation) within the same application. These can be called impact factors. For example in our case study, connection types, session types, and message types are impact factors affecting performance positively or negatively. There is confusion in term usage where a term sometimes refers to the nature of the requirement and sometimes refers to the design decisions. We will be using the term NFR to denote the nature of the requirement so a PIM metamodel is the place where we can define specific NFR types.

The difficulty of modeling and integrating explicitly NFRs (additional constraints) within the context of functional requirements is the fact that NFR affects the system as whole [18]. Non-functional requirements especially related to architecture are called quality attributes [4]. They affect design decisions where different quality of products can be distinguished. These are the decisions that drive the system architecture. The representation and categorization of non-functional requirements are still under research. More than one piece of information con- 
tributes negatively or positively to one NFR. Preliminary results show diversity in terminology and orientation [4]. In addition, there are dependency relationship among non-functional requirements. For example, in some cases maintainability requires portability. More importantly conflicts are found such as between performance and reliability as shown in our case study below.

The field of nonfunctional requirements as a component in requirements engineering is less developed than functional requirements[19], so there are only a few contributions such as $[14,20,21]$. We are going to follow a simple approach that would be compatible with E2EDE. For example, Zuh and Ian [22] proposed a generic UML NFR Profile, but it is not suitable to work under MDA because the assumption was to treat NFR and design decision in one place. These are different (separate abstracttion levels) according to E2EDE's principles. The 6-elements framework from SEI [21] follows a scenario-based approach that presents a good way to resolve the overlapping problem between NFRs. Our approach simply prioritizes NFRs to judge on design decisions, promoting automation.

Since the types of NFRs differ greatly among classes of application, a NFR Profile is needed as a domain specific language to allow system architecture to specify NFRs easily in a PIM metamodel. According to investigation in this track we have seen there is a need to priori- tize NFRs so the toolset can tradeoff between NFRs or resolve conflicts. Most of the current contribution to NFR considers the human factor and does not take account of tool support. For example Zhu and Ian [22] proposed for the relationship between design decision and NFR: support, break, help and hurt. Daniel and Eric [14] follow the same trend. In order to reach our goal we need to identity NFRs so the identifier concept is used to discriminate NFR instances. The 6-elements framework suggested by [21] could be a useful tool for Non-functional analysis at earlier development phases.

Figure 6 shows the elements of the NFR profile (Task C) used by an architect to specify NFRs which is specializing a metaclass class with two tag attributes. Below in the anchor is an example of an instance model. It also shows NFRs can be at Package level, which represents global NFRs such as Application Type, while delivery mode is at class level. It also shows that NFRs can act as Packagelevel, which represents global NFRs such as Application Type, while delivery mode is at class level.

\subsubsection{Transformation of informative PIM to PSM (Task E)}

The notion of transformation is hardly a new concept in software engineering. Traditionally, most software engineering work is conceived of as mapping, like the transformation from software specification to software design.

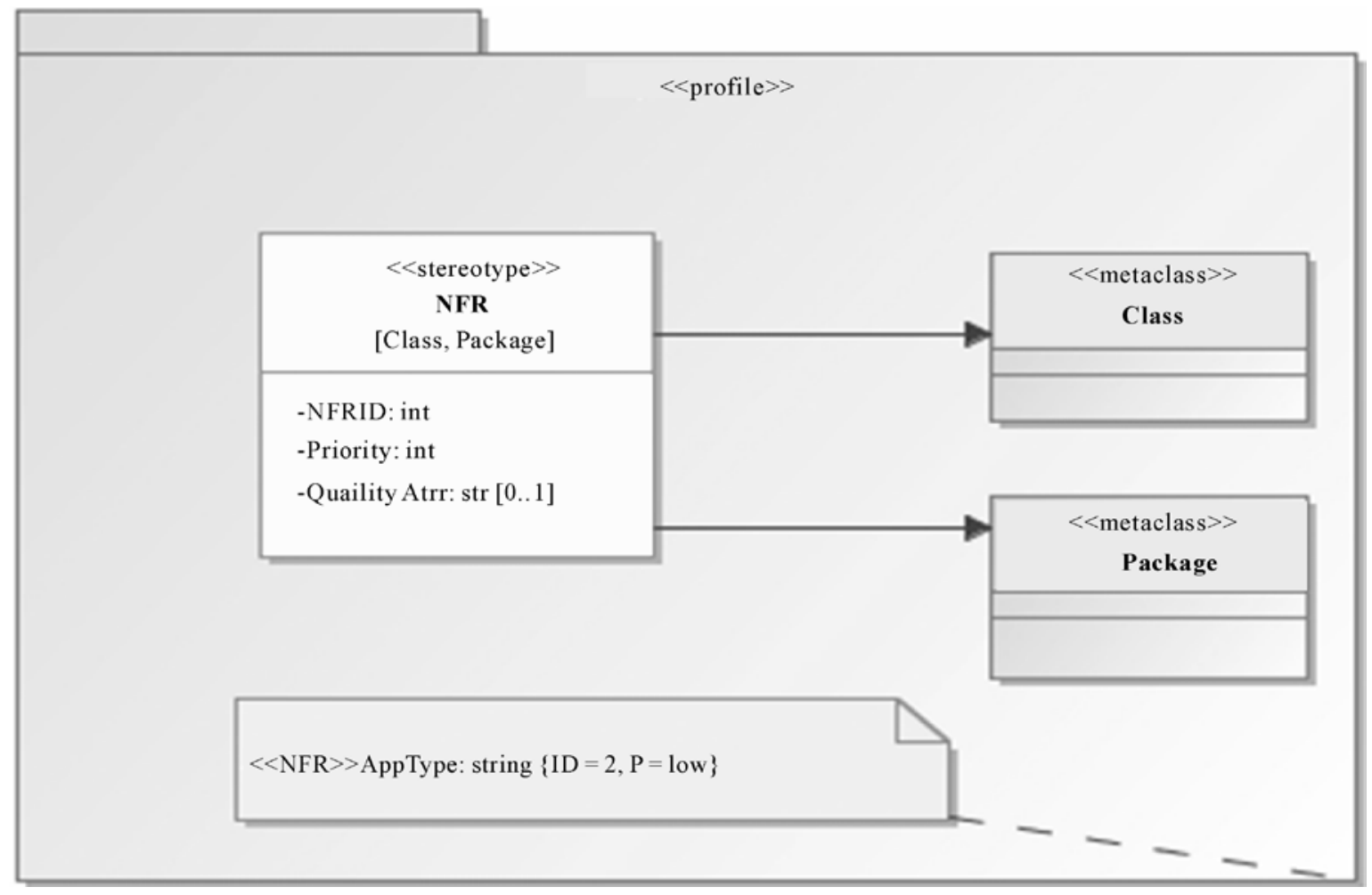

Figure 6. NFR UML profile. 
But what makes MDA different is considering mappings as first class objects in the effort to formalize this process by using standard languages such as QVT where mapping is from one metamodel to another metamodel. This approach is a prerequisite for the automation that MDA is seeking to achieve.

The key concept of MDA mapping is to resolve a structure pattern instance from PIM into a PSM corresponding structure pattern(s) instance. For example, in case of mapping from object model (PIM) to the relational model (PSM), a class instance will map to a table instance.

Our work proposes NFRs as major design drivers feeding the mapping process. This feature facilitates the mapping task where it becomes easier to select the corresponding PSM structure. NFRs make the difference between two PSM configurations. For instance, as we will see later there are two types of messages, persistent and nonpersistent, in the messaging system. If performance impact factor(s) are most important, the nonpersistent variant is suitable while if reliability is a design issue, the persistent variant is the best option. The former architectture will expose performance quality while the latter will expose reliability based on the additional computation the application needs to maintain the message storing process.

\subsubsection{Mappings of Class Operations}

The class structure in UML includes methods or behavior. Because maintaining different views in one model is com-plex, UML supports capturing dynamic behavior of the system separately by a set of behavioral diagrams such as the activity diagram. This subsection is about highlighting mapping problem from PIM instances of the process model to PSM instances of its process model. In this case a metamodel does not have user-defined operations, the MOF specification has defined default methods: create object, destroy object, and create link in each MOF metaclass [7]. Firstly, these methods are abstract methods. Secondly, mappings of structural patterns are somehow straightforward but the relationship between PIM behave- ioral model and PSM behavior is nonlinear. So when mappings for example occurred for attributes which are going to be columns in the relational model, there might be a set of corresponding operations (1 to M relationship) in the PSM behavioral metamodel for a corresponding class' methods in PIM behavioral model. However, there is no uniquely determined method to do that. Recent attempt for example in this case was suggested by [6], one possible MDA program written for medium-sized problem involving organizing a swimming meet according to FINA rules (insert and update native call) utilizing OCL capability to construct SQL PSM.

However, we can use the hierarchy structure of UML activity diagram to show the implementation of PIM me- thods in PSM as workflow as in our case study in Figure 7: Sendcase mapped to SendingProcess where it is extended into five operations as described in 5.3.

We notice here future work is needed to find a method that realizes the operations mapping so we can see how to incorporate variability and NFR concepts.

Generally the tasks comprise E2EDE process are shown in Table 1.

\section{Case Study}

A set of applications has been analyzed to produce the PSM architecture used by this case study as an implementation end. This family of products which are mentioned in Table 2 has exposed commonality in most aspects under messaging system domain. This analysis step is in line with the principle of domain engineering where at least three products should expose commonalities to justify the investment in core asset architecture [23]. A Help desk system is one example from this set of products which is a major component in most current business web-based systems. The idea is to allow a user to raise a case for some aspect which needs a reply from some organization web site party. An employee should consult through the same web site a list of cases or a specific case that has been presented as a request in order to update it. Then the update is sent back to user, who may be offline, as response. A broker is an intermediate module used to exchange messages between the system and users. Users contacting the system are durable customers. The nonfunctional requirement for system performance is higher than reliability.

\subsection{The Problem Specification}

The helpdesk software system is needed to service customer(s) and employee(s) at the same time. A customer will be required to insert identification information such as user name and password after registration-both of which will be sent for validation to the web site system which is located remotely somewhere on the internet. The customer as well as the employee will then be able to perform one or more operations.

The helpdesk must be able to provide the following services to the customer:

1) A customer must be able to login using his account information.

2) A customer must be able to submit a case to any employee linked to organization, by writing a text message and submitting it to a broker.

3) A customer must be able to view their case history (feedback), status, and details.

4) A broker needs to maintain a queue in order to schedule cases and differentiate between different users' cases. They also must be able to create a message. 


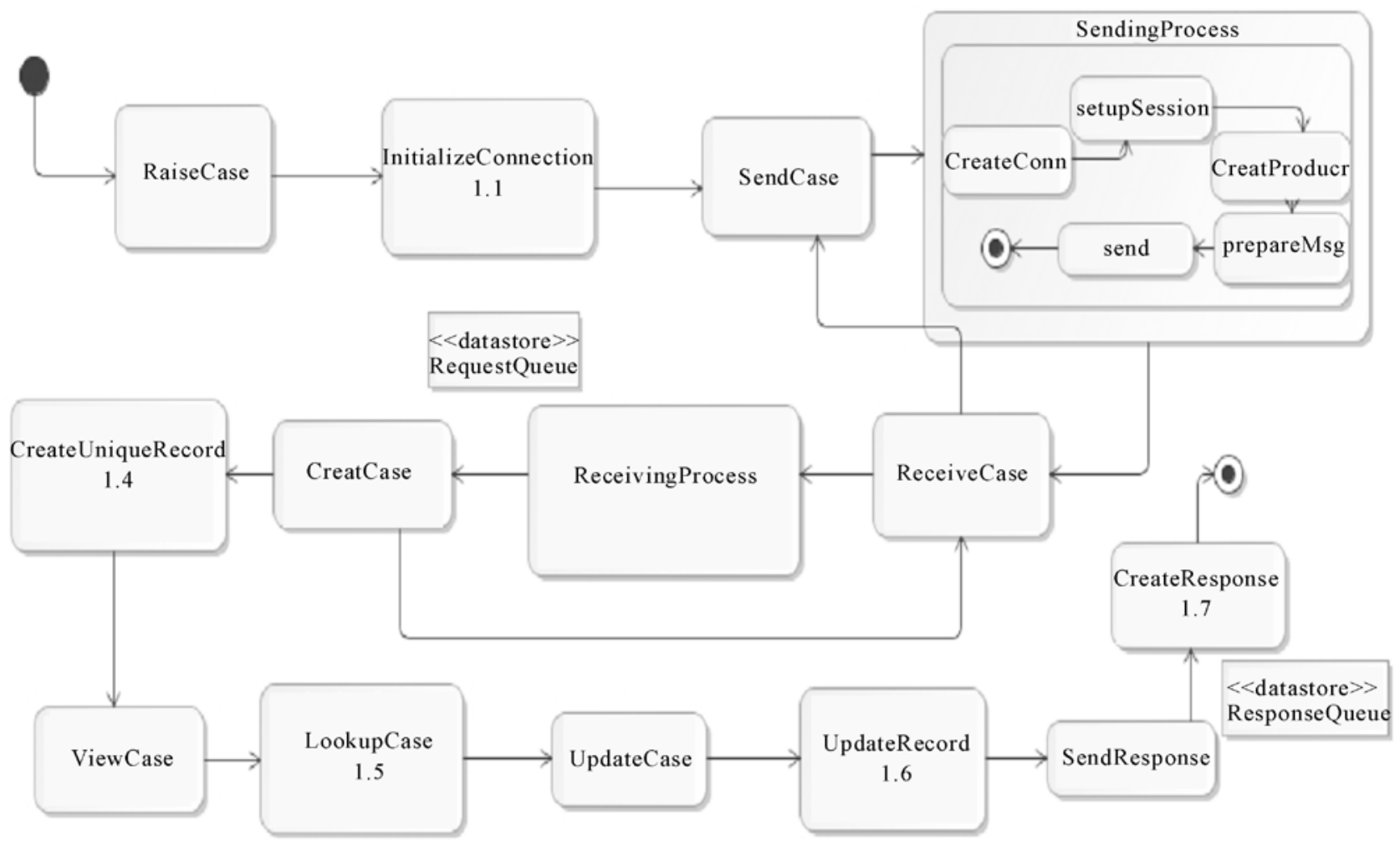

Figure 7. Behavioral mappings activity for helpdesk PIM to messaging system PSM.

Table 2. Configuration for a set of products from PSM metamodel with profile.

\begin{tabular}{|c|c|c|c|}
\hline Appliction domains & NFR Profile & Priority & $\begin{array}{l}\text { Variation point(Design Decisions) } \\
<\text { Message,connection,session,Ack }>\end{array}$ \\
\hline 1-Email System & $\begin{array}{c}\text { App-size }=\text { normal } \\
\text { Iscritical }=\text { yes } \\
\text { Delivery }=\text { notUrgent }\end{array}$ & $\begin{array}{c}\mathrm{P} 1 \\
\mathrm{P} 2+\mathrm{P} 3>\mathrm{P} 1 \\
\mathrm{P} 3\end{array}$ & $<$ persistence,queue,transacted, AutoAck> \\
\hline 2-Chat & $\begin{array}{l}\text { App-size }=\text { normal } \\
\text { Iscritical }=\text { no } \\
\text { Delivery }=\text { medium }\end{array}$ & $\begin{array}{l}\mathrm{P} 1>\mathrm{P} 2+\mathrm{P} 3 \\
\text { P2 } \\
\text { P3 }\end{array}$ & $\begin{array}{l}<\text { NonPersistence, } \\
\text { Topic,nontransacted, DupAck> }\end{array}$ \\
\hline 3-Forum & $\begin{array}{l}\text { App-size }=\text { normal } \\
\text { Iscritical }=\text { no } \\
\text { Delivery }=\text { medium }\end{array}$ & $\begin{array}{l}\mathrm{P} 1>\mathrm{P} 2+\mathrm{P} 3 \\
\text { P2 } \\
\text { P3 }\end{array}$ & $\begin{array}{c}<\text { NonPersistence,Topic,nontransacted, } \\
\text { AutoAck, }>\end{array}$ \\
\hline 4-Mobile application & $\begin{array}{l}\text { Reliability }=\text { high } \\
\text { Iscritical }=\text { yes } \\
\text { Delivery }=\text { high }\end{array}$ & $\begin{array}{l}\mathrm{P} 1= \\
\mathrm{P} 2= \\
\mathrm{P} 3=\end{array}$ & $\begin{array}{l}\text { <persistence,queue, } \\
\text { transacted, } \\
\text { FastAck }>\end{array}$ \\
\hline
\end{tabular}

5) An employee should be able view cases by individual case or list of cases and look for details.

6) An employee should be able to update a case.

This is the functionality needed to develop an application conceptual model (PIM) as we will see in Figure 8.

Figure 8 shows a class diagram for the helpdesk system. The basic structure of the class diagram includes six major classes: customer, login, broker, case, manager, and Queue with their responsibilities and relationships among them. In the case of the manager, one of the responsibilities is to provide access to a case in the response queue that has received a message from a broker and send the updated version back to broker; thus, Case, Queue, and broker have associations to manager. Case has association to the queue class. Case will be given unique ID and created so each case will represent uniquely an individual customer case which is stored in a queue either as a request if it came from the customer or response if it came from the system. The UI is specified in this PIM but we are not considering this part. It could be possible to capture an entire UI specification from this PIM that could be rendered by an outsourced third-party platform such as a browser.

We are using $<<$ NFR $>>$ stereotype to indicate non- 


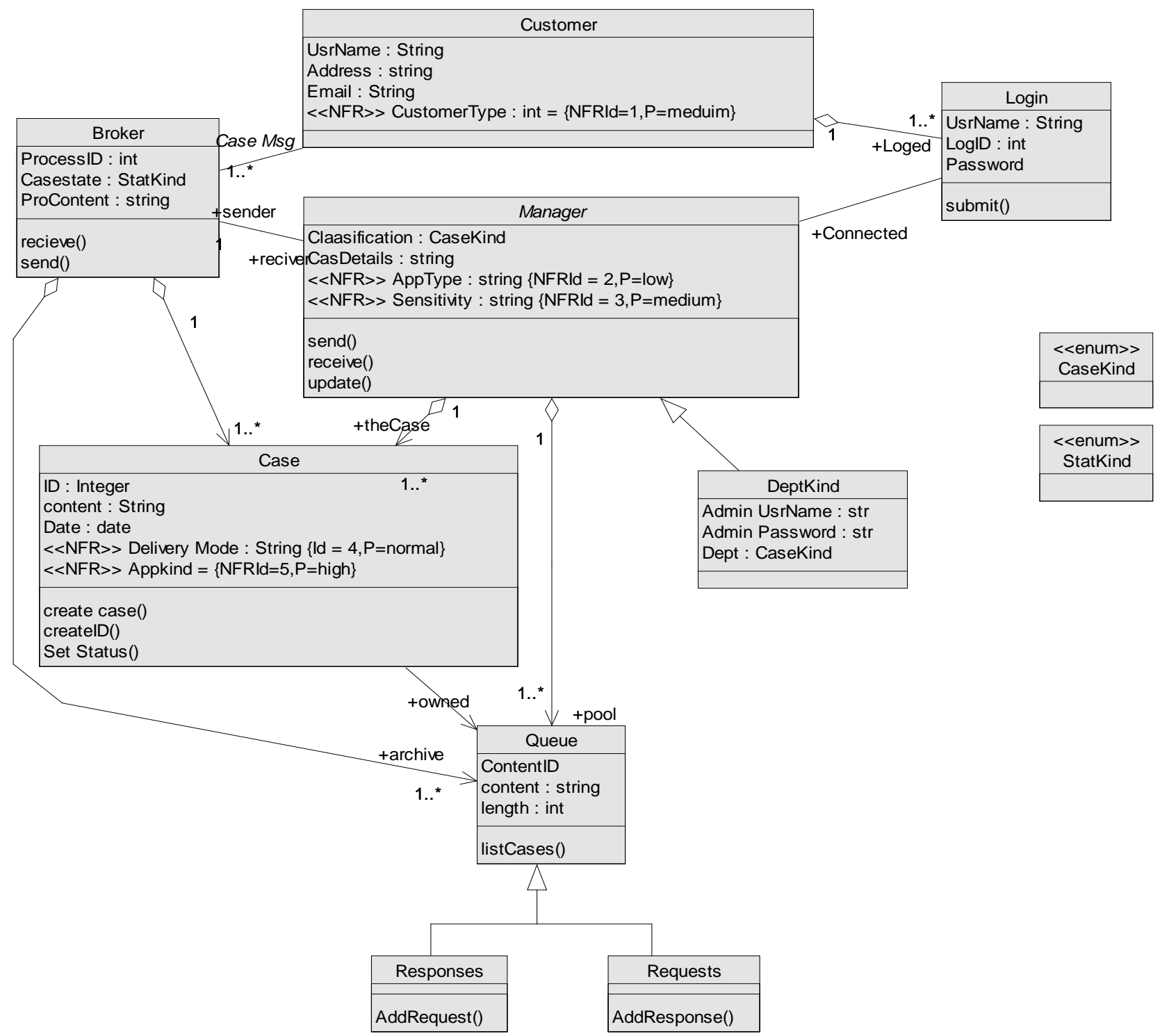

Figure 8. PIM: Helpdesk system with NFR documented.

functional requirements according to the NFR profile. Tag values are used to denote two pieces of information: ID to identify a NFR and priority (P) which assigns an integer number to indicate a priority level of NFR. These are the elements of the NFR specific-language used to model NFRs as described in 4.2.3 (Task D).

In the PIM shown in Figure 8 there are the following NFRs: Application sensitivity \{High, low, medium\}, AppKind transactional,nontransactional\}, CustomerType \{normal, durable\}, AppType \{normal, critical\}, and delivery Mode\{normal,guaranteed . (Task D). The interpretation of these NFRs will make sense when we link to design decisions as we will see later.

We now have a helpdesk system conceptual model describing functionality as well as nonfunctional require- ments.

The PSM in Figure 9 shows a messaging system which can be a realization of a PIM such as in Figure 8. It describes how data can communicate between two software entities: Producer instance and Consumer instance. A Session instance must be created between the two ends but to do that a Connection instance must be created first with suitable parameters. Session has two types as does Connection, while Transact means the underlying system should treat the data send as a transaction in database so the system should guarantee correct update, consistency, and can rollback a NonTransactional is to treat data not as a transaction. Consumer and data instances can be synchronous (Synch) or Asynchronous (Asynch) which refers to whether it is necessary or not for the consumer 


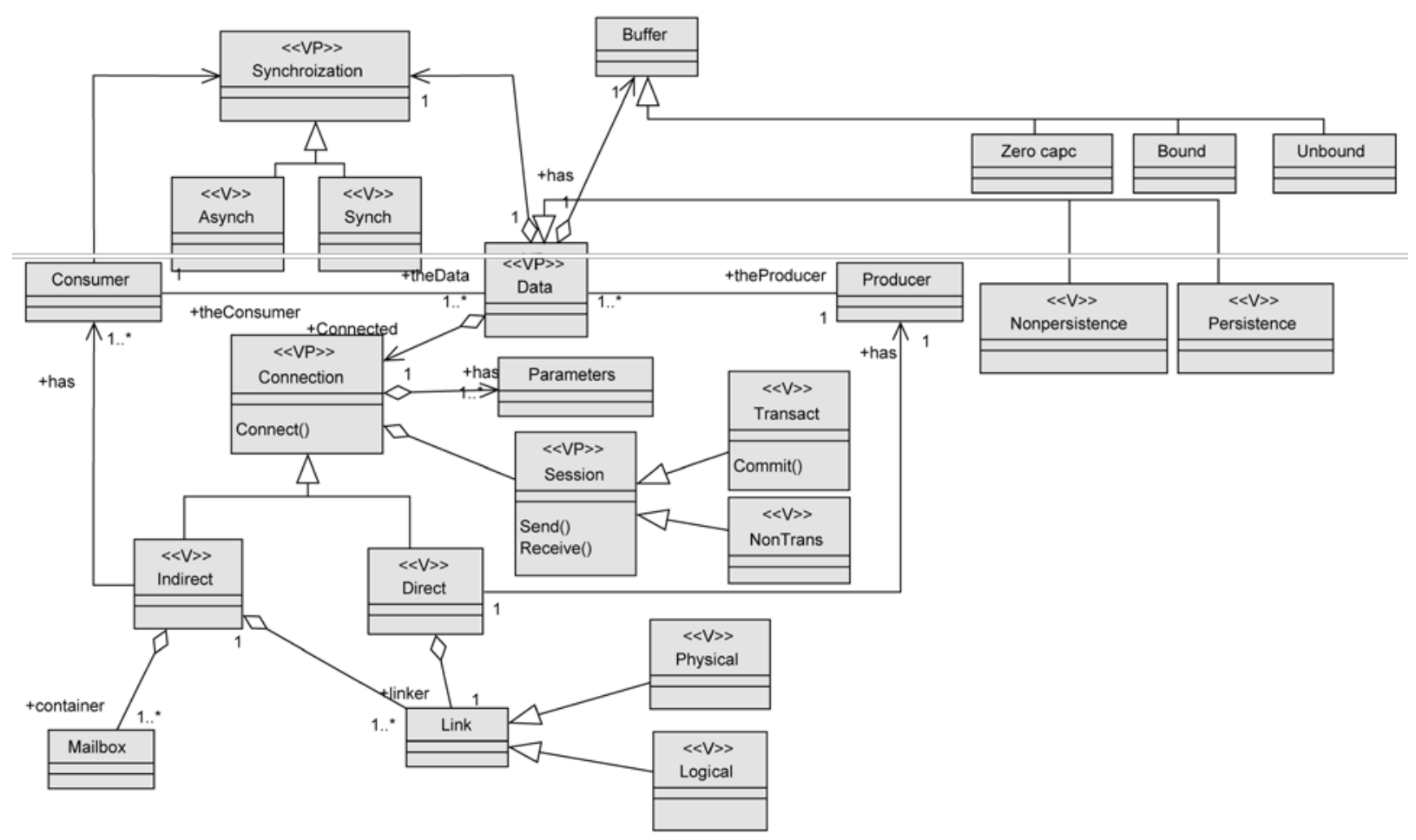

Figure 9. PSM of messaging system with variability in design decision.

to attend at the time of connection. Connection is Direct type or Indirect type transaction in case of failure.

We can call Queue for the former which allows one receiver per send action and Topic for the later that allows multiple receivers per send. Data can be Persistence type which means using backing store, preserving it in the case of any failure, or it can be NonPersistence. (Task B)

We notice this abstraction deals with concepts that are from implementation space not like the PIM concepts that are from an application domain.

Figure 9 shows the capability to deal with different design decisions configuration that are represented by variability (Task A, C). For example Connection is denoted by $<<\mathrm{VP}>>$ stereotype which can take Direct or Indirect variants. Likewise session denoted by $<<\mathrm{VP}>>$ with its two different variant kinds. The focus on the discussion will be only on variability that is non-functional with respect to the PIM.

\subsection{Mappings (Task E)}

In order to explain how to apply this principle we will describe mappings of this case manually, while it should be automated. The NFR in the PIM of Figure 8 will guide the mapping process to configure a suitable PSM from Figure 9 automatically. For instance, the two data variants: persistence or NonPersistence is selected according to the application need for reliability or performance so delivery mode will determine that. The current case study says Application Sensitivity is not high and a delivery mode is normal hence these two NFRs are not reliability factors which require maximizing performance. The delivery would use NonPersistence option which does not necessarily insure message delivery by for example storing message until a receiver becomes available.

Similarly, the Session variation point has two variants: Transacted and Nontransacted. Because the AppType (i.e. size) NFR is normal application in the help desk PIM Nontransacted variant is the most suitable.

This factor affects performance directly for example when transacted variant is used performance is impacted negatively compared with the Nontransacted mode. This is because there is additional overhead for resource manipulation needed in the system for transaction mode.

Connection variation point has two connection variants: Direct and Indirect which means single receiver versus multiple receivers per message. They are selected based on AppKind and CustomerType Non-functional requirements. A Transactional application, for example banking transactions, requires usually direct connection such as for doing funds transfer. The same is true in our case study where direct connection is selected for request and response messages because CustomerType is single user. In contrast, mobile applications such as advertisements prefer broadcasting a message to group of receivers so indirect connection is the most suitable variant. In that case customer Type can be multiple users. 
Generally in terms of performance indirect connection is contributing positively while direct connection is contributing negatively. The same message is forwarded to different subscribers which mean lower resource consumption.

Note that we need one of the variants to be set as default because the PIM and PSM are independent so that the default will be selected in case there is no corresponding NFR(s). For instance Figure 10 shows that the default variant is selected ([2]) for ConnectionVP if there is no corresponding NFR addressed. We notice also two or more design decisions determined by single NFR such as Appkind can be used to decide both connection and session variants.

Table 2 shows informally part of mappings from Helpdesk system PIM to messaging system PSM with NFRs guidance. It also shows how application concepts turn into design concepts. For instance the Case from PIM metamodel which is a unit of work between customers and company holding the necessary information will be mapped into three objects: Message, Connection and Session.

Because we have two kinds of messages and so two different bodies of computations, we need to judge on suitable design by looking into NFRs presented in the PIM according to for example performance or reliability.

NFRs can be extracted from system specification by different formats and meaning. For simplicity Iscritical (such as OCL style) is used instead of AppType. The mapping rules that can be used to implement the mappings specified in Table $\mathbf{1}$ are shown below.

PSMR $=$ PSM repository holding instances

$\mathrm{Pi}=$ Priority

[1] CaseToData

IF (Iscritical=Yes) and (delivery=guaranteed) then

If (P1[Is critical] $>\mathrm{P} 2$ [delivery])

Then store in PSMR ([Data,type=Persistence)])

IF (Iscritical =NO) and (delivery=guranteed)

Then store in PSMR ([Data,type=NonPersistence)])

IF (Iscritical $=\mathrm{No}$ ) and (delivery=normal)

Then store in PSMR ([Data,type=Persistence])

[2] CaseToConnection

Select connection.default-V [Queue in this case]

Store in PSMR ([Connection.type=connection.default-V])

[3] CaseToSession

IF (AppKind.value=transactional)

Then store in PSMR ([session,type=transacted])

Figure 10. Part of informal mappings rules from helpdesk PIM to messaging system PSM.
Figure 10 like pseudo-code shows a sample of mappings rules to transform PIM instances with existence of priority consideration into PSM instances. For example, [1] describes a conflict situation where if the application Iscritical and at the same time the delivery is guaranteed, the selection of design decision will depends on the highest priority. In this case persistence (factor of reliability) is chosen because P1 is greater than P2. Note here priority is used only in the case of NFR values causing a conflict.

We notice by this way an application could be configured at the two extremes: reliability and performance using suitable design decisions represented in the design artifact with NFRs guidance. It is also possible to configure an application in between these two extremes. Thus our method affords different products with architecture designs at different levels of quality-attributes. Inputs for mappings will be PIM metamodel (holds application instances), NFRsrepository (NFR instances), PSM metamodel, and Variability (design decision instances).

\subsection{Class Methods Mapping}

This activity is intended to realize the abstract operations expressed by one kind of behavior diagram for the helpdesk PIM metamodel. We can use an activity diagram to show the control flow and instances creation during the execution of the mappings from PIM to PSM at this stage. For example in the behavior instances model of Figure 8, the sendcase method in broker needs to map into the following sequence: createObject (connection), createObject (session), createObject (producer) as in the behavior instances model shown in Figure 7. It shows the control flow of mapping activity and relationship occurrences between source (helpdesk behavior model) and target (messaging system model). For example, raise case will be mapped to initialize Connection and sendcase will be mapped to sending process. We can determine PIM (source) and PSM (target) actions from this activity diagram. For example PSM activity are, initalizeConnetion, SendingProcess, ReceivingProcess, CreateUniqueRecord, lookupcase, UpdateRecord and createResponse (from 1.1 to 1.7$)$. But still as we mentioned previously more investigation is needed in this place to map a PIM process model to PSM process model and understand this mapping completely.

\section{MDA in the Context of Design Pattern and SPL}

It is a claim in this paper that MDA is a re-use approach. In this section we see how MDA can fit in with other common re-use approaches such as design pattern and software product line (SPL).The investigation of this relationship is the reason behind approaching E2 EDE. 
Varibility and Nonfunctional requirement concepts are borrowed as we have seen in section. MDA is a special case of design pattern techniques as we will argue in Section 6.1, while MDA and SPL have a synergistic relationship according to observations described in Section 6.2 .

\subsection{MDA and Design Pattern}

The design pattern concept goes back to Christopher Alexander [24]. His definition identified a relationship between three parts constituting a pattern: problem, solution and context. In software engineering, a design pattern is a general reusable solution to a commonly occurring problem in software design [25].

\subsubsection{Limited to Domains with a Well-Established Code Base}

The nature of solution provided by MDA is more specific to the problem domain than the design pattern which is more general because there are many kinds of design patterns [26]. A general purpose pattern perspective in solving problems is more expensive in terms of the establishment of working environment than in MDA, which is characterized by its well-established specific backend. Typically, MDA is used to target platform(s) that have already been crafted. For instance, large scale software RDBMS (a complex proven solution) can be utilized automatically by tools which transform PSM relational model after mapping to the SQL language. In contrast, for a pattern to be executed generally involves establishing new tools. For instance, Yacoub, Xue and Ammar [27] proposed their own visual systematic tool.

\subsubsection{Separating Concerns Allows Application Logic and Platform to be Variable and Encourages Re-Use}

It is observed that design pattern tends to integrate the behavior aspects with implementation aspects which result in risks of platform changing or volatility. Further, some implementation details become suppressed as consequence of behavioral variation as in the publish-subscribe pattern which does not say anything about remote objects design [15]. If this pattern is used in a distributed environment it becomes necessary to distinguish local from remote objects which is not available as a design decision at design time.

\subsubsection{End of Pattern Life Cycle}

Design Pattern follows a life-cycle as patterns become more mature and quality increases [28]. MDA produces high quality patterns because PSMs are end of the pattern life cycle. Although the nonfunctional requirement emerged first in the design pattern approach, MDA gives a wide opportunity to represent NFR explicitly. It is the critical requirement that discriminates between pattern architecture designs. In fact, it is still a research question how to graft design pattern with recognition of NFRs. In Buschmann [15] we can observe the role of NFRs in balancing design forces.

\subsection{MDA and Software Product Line (SPL)}

Software product line engineering is a paradigm to develop software applications (software-intensive systems and software products) using common platforms and mass customization [2]. The intended goal is to avoid reinventing the same solution for different applications in order to achieve shorter development time and high quality products (i.e. Nokia mobile applications). There are two distinct development processes adopted by SPL: domain engineering and application engineering. The former is concerned with design for reuse by seeking communalties and variability in the software product line. As a result a reference architecture called product line architectture (PLA) is established. The aim of the latter is to drive applications by exploiting the variability of the software product line.

\subsubsection{Defining Variation Points and Variants}

The central concept in SPL is the explicit representation of variability. Variability is a variable item of the real world or a variable property of such an item [16].A variant identifies a single option of a variation point and can be associated with other artifacts corresponding to a particular option (dependency relationship). For example, payment method as a variation point can be realized by variants: payment by credit card or payment by cash, etc. It is necessary in SPL to identify variability by defining variation points and variants, which is used by a selection process to produce different products.

There are two types of variability: Variability in time, which is different versions of the artifact at different times (i.e. performance), while variability in space refers to an artifact in different shapes at same time. For example "system access by" variation point in a home security system can have two variants: web browser and mobile phone. Variability in space is the central challenge faced by SPL, so management of variability is the main issue in this engineering approach [16].

A set of closely related objects, packaged together with a set of defined interfaces, forms a component [28]. Usually a component-based approach is used to realize SPL concepts.

SPL tightly couples application and implementation models together.MDA as an approach reduces the SPL to abstract computational processes. It separates the application from implementation by creating PIM and PSM abstraction levels. 


\subsection{MDA in the Context of the Software Product Line}

Both software product line engineering (SPL) and model driven architecture (MDA) are emerging as effective paradigms for developing a family of applications in a cost effective way [3]. SPL through its feature-based models provides a capability to capture variability in intensive systems, while the effectiveness of MDA is primarily due to potential for automation it offers for variability in technology. Generally MDA can fit into SPL as an effective software development method. For instance MDA can tackle implementation variability within a specific platform. So the synergistic relationship between the two approaches has been studied recently $[4,20,29]$. The basic differences between the two approaches are as follows:

\subsubsection{MDA Decouples Implementation Model from Application Model}

The PSM is constructed as an API to specify the implementation aspects for an intended target such as relational database model. Similarly a PIM model is built which specifies the business logic. This will add value by enabling MDA to tackle technology variability which allows the same PIM to be rendered into different platforms or PSMs.

Although components raise the reuse level a little bit, they still suffer from the software evolution problem. For example, any small interface changes will entail finding everywhere the interface is used, changing it to reflect the new interface, testing new code, reintegrating and retesting the system as whole. Therefore, a small change in the interface can cause enormous changes by following each code part that refers to this component interface. In contrast, the PSM, an intermediate subsystem, abstracts this tedious task by concentrating the changes in one place. Also, MDA avoids the problem of features explosion that tends to complicate maintenance [9]. In addition, keeping a mapping function separate avoids swamping the source model (application) with implementation details and reduces the problem of model divergence because the target (implementation) is generated [29].

Furthermore, MDA increases architecture longevity (ageing) compared to the fact that sometimes PLE suffers from architecture lifespan which may reach end of life [22]. In this case evolving architecture will be expensive or risky. MDA's potentiality comes where evolving the architecture becomes much cheaper because each of PSMs and PIMs are adapted separately and they do not carry any volatility risks (technology variability).

\subsubsection{MDA is Intended to Automate the Craft of Code}

The potential of MDA is due to the capability of automa- tion it offers. It is recognized that if we will be able to formalize the model to the extent that it has no ambiguity and the model is machine readable (executable) then the code in principle can be mechanically generated. MOF is a powerful metamodeling language that realizes this trend by allowing tools to interoperate and accurately modeling the conceptual model of a design language such as UML. Crafting code becomes a model driven process wherein a transformation from source model (PIM) to a target model (PSM) can be automated by for example QVT tools. Eventually the PSM can automatically mapped into text (code). MDA works best if the scale of PSM objects is the same as that of PIM. The mapping function is kept separate so that it can generalize some concepts and it can be repeated many times (repeated design decisions) showing a big picture of reuse. The mapping function can be automated at the instance level because it is an algorithmic process in which generic transformation rules are established at the type level. The general feature of automation is the synchronization between the two ends.

\subsubsection{Higher Abstraction and Systematic Development Methodology}

The main goal of MDA is to raise the abstraction level higher than traditional middleware and 3 GL/4 GLs programming languages. This means taking advantage of software-platform independence that enables a specification to execute on a variety of software platform with a stable software architecture design. The granularity of code re-use will increase to the level of a PSM (ADT) instead of components as in SPL. The PSM is scoped to this level of code reuse. For example relational database PSM is an abstraction for the family of relational databases above any specific technology. Also, there is a difference between MDA and SPL in defining interfaces to components and frameworks via an API. In MDA, the interface is not concrete but it is meta-interface exported by marking models [29]. The mappings are externalized and generalized, which can be reused in similar problems.

MDA is standards-based development method which is specified entirely by a nonprofit organization, OMG, since 2001 [1]. It involves algorithmic mapping processes from model to model (PIM-PSM) and from model to text (PSM-code). The mapping process is rule-driven in which transformation rules are expressed by a standard language (e.g. QVT). However, different viewpoints could be constructed for different abstraction levels. Formal mapping functions will often fill the gap between any two different abstraction levels (consider compilers). Further, having MOF as metalanguage and other well-established OMG standards (i.e. XMI), it promises industrial-scale systematic re-use and integration capability. 


\section{How Design Pattern and SPL Contribute to E2E2D Engineering}

The survey of the relationship among MDA, SPL, and Design Patterns has shown a synergistic relationship. MDA improves each approach by supporting these quailties: automation, proper management of technology changes or volatility, high granularity of reuse and more important a capability of integration.

Design Pattern is not an end to end concept because it is an abstraction for software implementation.

Design pattern could be used to construct the architectture in E2EDE. It adds value by acting as a proven solution and a documented experience.

SPL is an end to end concept but in addition to the problem of coupling application and implementation together, it does not tackle the variability in the implementation part. In contrast, E2EDE is mainly addressing this challenge. In addition, there is no concrete link as in E2EDE between higher level models and lower detailed models.

SPL engineering gives another insight for E2EDE: the concept of explicit variability representation and management. Introducing variability explicitly in the PSM helps mainly in its construction. This means a PIM can become informed about variation points that are documented explicitly therefore it becomes possible to automate the design decision process. A UML profile for specifying PLA [3] can fill the gap between PSM and the PLA core assets artifact.

Metamodeling and MDA are an alternative technique successfully used to organize SPL and feature model concepts as demonstrated by Muthig and Atkinson [11]. Furthermore, unlike orthogonal models, the variability model and original model would not be separated, which increases readability.

\section{Strategic Messaging System PSM}

The philosophy of MDA is to do more investment on metamodels so as to hopefully obtain payoff at production time by producing larger number of products. It can be conceived as the same scale as where database systems and X11 [20] are considered viable.

We have looked at PSM in Figure 9 in the previous section as a specific implementation for helpdesk system. In fact this PSM was built from a general messaging system perspective. The concepts in this PSM form an ontology. There are many messaging systems which commit to that ontology.

Examples are: Chat system, Email system, instant messaging system, media streaming system, mobile applications, etc.

As we argued before re-use is a major trend in the software development community. Important are not only reusable components but also strategic reusable assets like models and transforms.

Table 2 shows a simple configuration for four products as a picture of the benefits of re-using the messaging system PSM. Further, it is obvious that the rationale of this specific architecture design does not exhaust the E2EDE approach. An architect can reason about different architecture designs.

In Table 3 we see there are number of NFRs common to this set of applications, which are re-used to make a design decisions. They are App-size (i.e. Application size), Iscritical and Delivery. Both App-size and Iscritical are a kind of Package level NFRs while Delivery is a class level NFR because it is about an object class inside the system. Design decisions are: message (data), connection and session, and Acknowledgement mechanisms. In the example of email system two reliability factors are higher than the other; App-size has lower value than for delivery and apply-size, therefore message (data) delivery is persistence with Queue type connection and transacted session. The Acknowledgement will be given normal value which is AutoAck. All these values makes reliability higher than performance because of the overhead processing (i.e. store) which what is said by NFRs. The inverse

Table 3. Part of mappings from helpdesk system to messaging system.

\begin{tabular}{|c|c|c|}
\hline PIM & Relevant NFR & PSM Variation Points \\
\hline 1- Case & $\begin{array}{l}\text { Data needed between producer and consumer and let the system works so } \\
\text { it is functional. But there is a quality on its processing based on } \\
\text { priority and type. }\end{array}$ & $\begin{array}{l}1.1 \text { Message } \\
1.2 \text { connection }\end{array}$ \\
\hline 1.1 Data [message] & $\begin{array}{l}\text { Apptype }\{\text { normal,critical }\} \text { or sensitivity }\{\text { low, high }\} \text { and } \\
\text { delivery mode }\end{array}$ & $\begin{array}{l}\text { 1.1.1 persistence or } \\
\text { 1.1.2 nonpersistence }\end{array}$ \\
\hline 1.2.x.1 session & AppKind and delivery mode & $\begin{array}{l}\text { 1.2.3 transacted } \\
\text { 1.2.4 Nontransacted }\end{array}$ \\
\hline 1.3 broker & User or customerType & $\begin{array}{l}\text { 1.3.1 Consumer::Asynch Or } \\
\text { 1.3.2 consumer::Synch }\end{array}$ \\
\hline
\end{tabular}


of this situation typically is in Chat and Forum application where P1 of application size put into highest priority than data delivery and Iscritical so the configuretion of parameters is set to increase performance. The mobile application comes in the middle between performance and reliability more oriented to reliability. Note that this is an arbitrary configuration but any other scenarios are possible. The point is by that we can see an example of NFRs and variability reusing among products in messaging systems.

\section{How MDA Works}

MDA is new trend in software development. This section sketches key points about MDA implementation.

The history of software engineering shows that a software design model is a complex object that needs to be maintained during a project life cycle and refined over a long period. CASE tool (computer-aided software engineering) is used to allow easy model creation, editing, rendering etc. In this case, a tool designer utilizes information system technology to keep this complex object in a database called a repository. A repository consists of a schema which stores model instances [6]. In fact this repository does not need the complete commercial database machinery. There are recently emerging MOF-standards like XMI [30] used as a mechanism not only for persistence purpose but also as a mechanism for exchanging models between tools which it was difficult before in a classic CASE tool (i.e. magic draw, rational rose). Many recent MOF-based toolsets support in addition to efficient access methods, both system and user- defined API serialization mechanisms in which developers can render a model using an XMI concrete syntax for different purposes. There are many tools with different features and capabilities working in this context, extensively studied in [31]. EMF [32] an open platform adopting MDA principles provides a Java code-generation facility to achieve interoperability between applications based on a MOF meta-modeling framework.

\section{E2EDE Implementation}

The implementation of E2EDE need to be considered as there is some limitation in current MDA tools. Our approach in this space is to separate working on the model view from the implementation view the same way UML gives a different views for different purposes such class diagram and activity diagram.

The proposed profiles are useful in terms of readability and explicit showing of the NFRs and VPs but for implementation it needs suitable representation to fit the MDA computation environment.

There are three reasons underlying the solution suggested in this section: source, target and mapping meta- models. Firstly, current tools have a limitation of recognizing a profile instances in a model annotated by a profile elements such as MediniQVT [33]. (Tag values are not visible to QVT pattern expressions.) Therefore we suggest a representation for profiles to resolve this issue. Secondly, if we look practically to the mapping the metalevels concept breaks down when we compare two systems. For instances, if we used UML as PIM metamodel and MOF as PSM metamodel, the mapping is from instances of M0 objects to instances of M1 objects. The same is true more generally when we use Profile instances that are at level higher than instances level of the metamodel. In our specific case, profile instances are at M1 level while the metamodel instances needed by QVT engine are at M0 level. However, OWL-Full [34] can be suggested as an alternative technology to UML which could resolve this solution. OWL has an OWL metaclass class which is itself a class, so we can build a profile mechanism by declaring subclasses of OWL class.

Finally there is a need for linking a single VP with a set of NFRs and mapping variability should be considered. (Task G)

Figure 11 describes the relationship between VP, NFR and a Design Decision. A design decision is one of two kinds: selection which denotes the normal variability exists on PSM, and compiled which represents the mapping variability highlighted in the previous sections. This sort of design decision groups related rules that have some common property which is modeled by the attribute rank. An instance of compiled design decision is associated with an instance of NFR because NFR(s) is the reason behind this grouping.

For example, consider how the mapping variability discussed in Section 4.2.2 could be represented. Also, more information details about design decisions can be added, for instance to compiled subclasses, like the effects and cost of effect etc. However, an instance of a design decision is an opaque rule specifying the creation of valid PSM instances when its precondition is satisfied as shown in the following. A program manipulating this metamodel should differentiate between three modes: default, application of a rule, and conflict resolution. A conflict mode needs to refer to NFR's priority. Seduocode based in QVT relation language is shown in Figure 12 .This part showing application of Task F.

Figure 12 demonstrates statements describe two disjoint types of connection that will only be created as PSM instances when certain Preconditions are satisfied. The function of the Guard Predicate class is to collect VP related NFRs which has multiplicity one to many. This means a pattern structure in PIM will be linked with one variant through one or more NFRs. For instance, in the two examples we have two sets of NFR related to Direct 


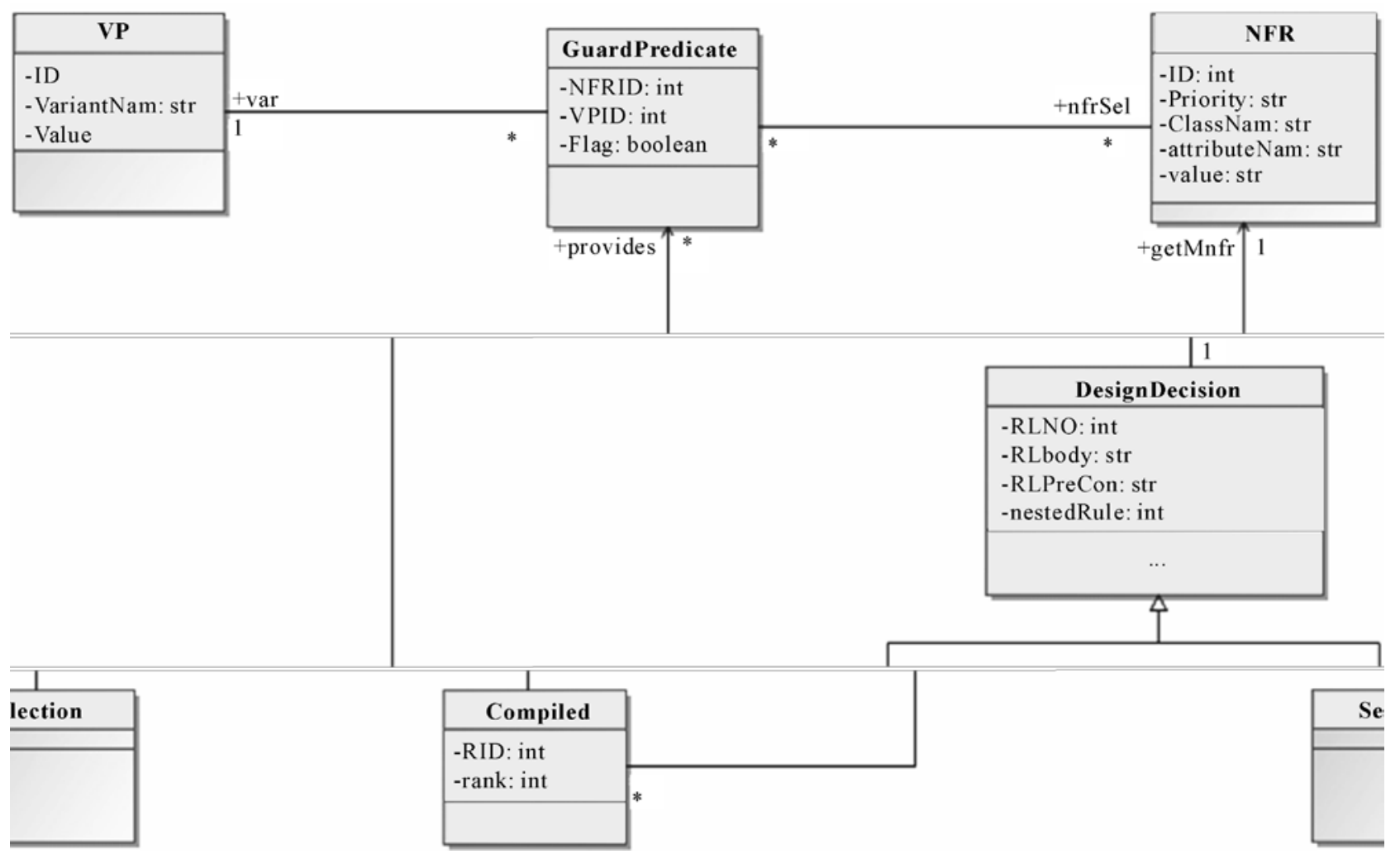

Figure 11. PIM to PSM mapping metamodel.

1-RLPrecod = CustomerType =important,

AppKind=transactional

Enforce domain MSGPSM co:Conection \{type='Direct',

name $=$ con $\}$

2-RLPrecod $=$ CustomerType $=$ normal, AppKind=Nontransactional

Enforce domain MSGPSM co:Conection \{ type='Indirect', name $=$ con $\}$

Figure 12. Opaque rules for mapping variability rules.

and Indirect variants respectively: \{important, transactional\}, \{normal, Nontransactional\}. Note that variants in PSM are disjoint and covering because they are alternative design elements. NFR and VP are imported from corresponding packages.

The metamodel in Figure 13 is a lightweight UML2.0 metamodel used as an example by the QVT specification. We use this as a base for presentation purposes (Profile). The full work makes use of UML.

The extension or adaptation to this existing metamodel was special NFR (SNFR) metaclass, general NFR (GNFR) metaclass and NFR metaclass. Working with this case study shows that there are two kinds of NFR: package level (general) and class level (special).

An extension to the same UML simple metamodel could be done for variability model using an extension to the metaclass class to represent variation point, variant and QuailityAttribute. The same extension is found in the literature such as $[2,3,12]$ but there are two problems with this. Firstly, it does not model mapping variability and for example the conflict cases that arise when we link NFRs with variants. Secondly, it is impossible to use the UML toolset to do that modification because it is at the level of UML metamodel. Here the proposed approach generally involves Profiles, packages and model manipulation.

The meaning of variability in PSM is somehow different from traditional variability in SPL. In E2EDE, variants are disjoint and covering which represents only alternative design decisions. These decisions can be overlapped and not covering in SPL. Variants in E2EDE exist on a PSM artifact to represent Nonfunctional while SPL traditionally represents only functional variability. There is no dependency such as between VP-VP because it is already inherited from the UML design language.

\subsection{Packages (Task G)}

PSM variability needs to be represented in a way accessible and without ambiguity to the relationship programs. 


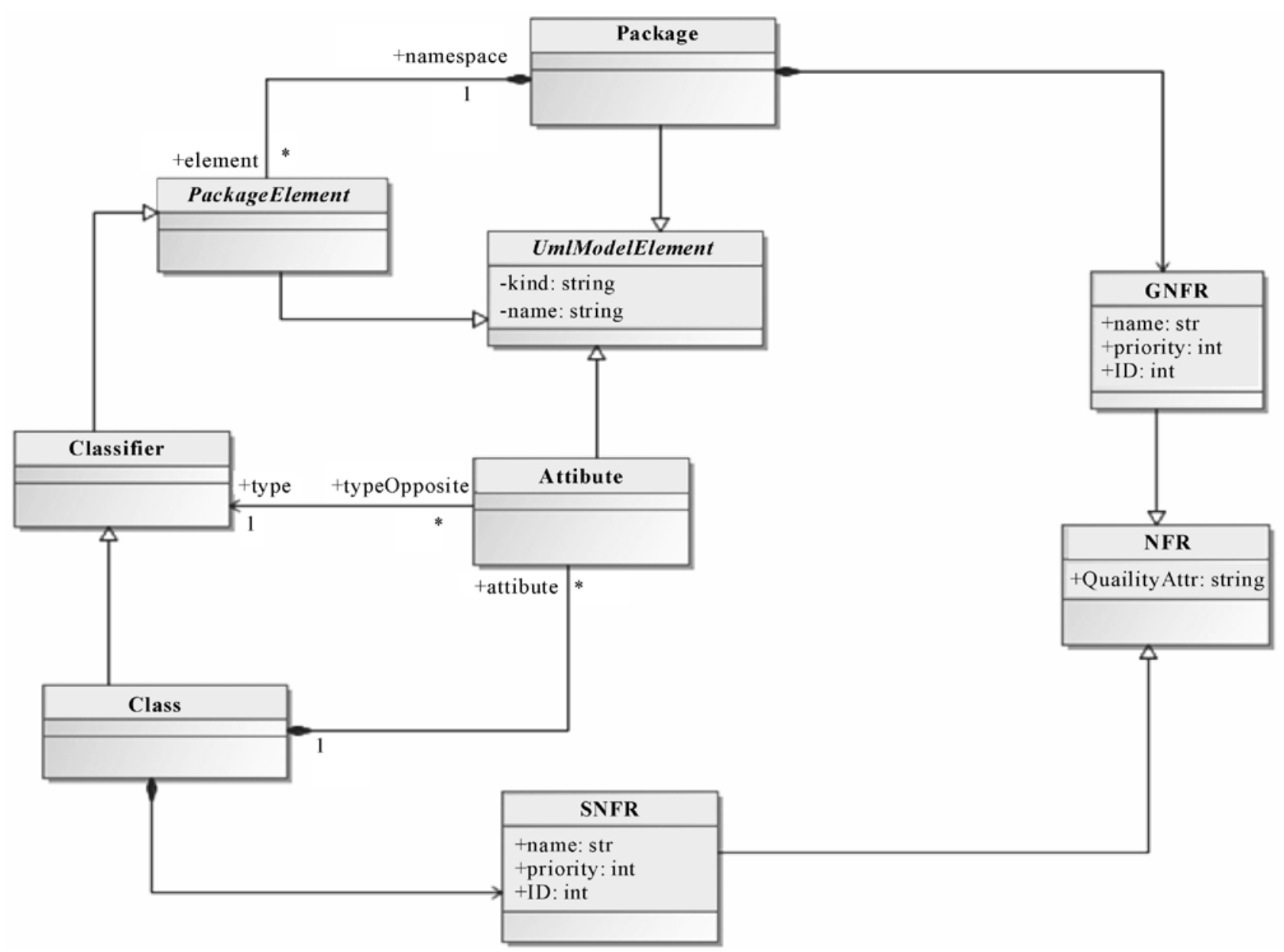

Figure 13. Simple UML2.0 metamodel extention from QVT specification as Profile implementation.

The relationship program has end to end functionality. It is intended to link a PSM variant with the relevant NFR(s). The traditional mechanism in literature used to model variability is through a subclass structure of the UML class model like [12]. This is suitable for humans but if the system is scaled up, it would be difficult for a human to comprehend that system. The second problem is that some times in these large system names of classes, properties, and association etc, can be ambiguous. Therefore we need a representation mechanism that allows the program to find model elements. MOF and UML support a Package mechanism which has a capability to make names of members unique within the package that owns them. Further it is has a facility to disambiguate names where necessary by adding the package name as prefix. So both human and programs could easily access model elements without ambiguity. Further a package may need to import or merge another package.

Therefore, the semantic operations of incorporating a subclass (variant) in the model will be through legal standard package operations.
In UML2 infrastructure a package [35] is defined to group elements, and provides a namespace for the grouped elements. A package merge is used as basic re-use mechanism that allows extension by new features. For instance, UML2.1.2 superstructure builds on the Infrastructure library by re-use of the kernel package. It is defined in UML2.1.2 infrastructure as a direct relationship between two packages that indicates the contents of the two packages are to be combined. Conceptually this means there is a resulting element combining the characteristics of both source and target.

Since we modeled VP and V using the generalization concept, a subclass is always an extension for superclass i.e. by adding new structural features. A package merge has these capabilities. Therefore, a PSM super-subclass structure will be modeled using packages.

The second value of using a package is that it is a powerful mechanism for embedding an entire metamodel (sub architecture) to represent a variant that could be reused in the main model (namespace). It is effective due to its capability to represent PSM implementation varia- 
bility that can scale up as practically used by OMG as a basic building block to develop and reuse a variety of infrastructure and superstructure constructs. Further the capability of package operations (i.e. import, merge, etc) allows one to build complex structures by combining simple constructs using a systematic rule. This feature in the programming languages concepts is recognized as orthogonality [36].

So now we have the representation of variability in PSM using the package mechanism. In addition, we have NFRs represented in PIM metamodel which has representation in Figure 13. They were two kinds: package level NFRs and class level NFRs. To this end we need a relationship program using NFRs to select the suitable variant(s). This will be modeled using model manipulation tools. But in order for this relationship program to work we represented elements of the problem in way easier for the programs to find and manipulate (Package).

\section{Lessons \& Realistic of E2EDE}

The key point from the step toward strategic PSM like the one presented in section 8 is since there are a group of different products complaint with a standard interface, they are sharing an abstract data type (ADT). It becomes easy for example to replace one by another, for example Dell laptops standard architecture is the reason behind a wide set of products. Another example from our community is the service-oriented architecture where its standard interface leads to proliferation of applications and what is known as Agility. This scenario even could be applied to situation where there is no standard specification. Here we need a reengineering process to fill the gap but this time with the lowest reengineering cost, with assumption that the different products have largely similar functionality. Typically any drift from common functionality would be resolved as a mapping from the PIM to the PSM. Any further changes made necessary by use of a particular platform should be relatively minor.

Typically it is the case of messaging system PSM there is no standard specification but E2EDE encourage reaching agreements on messaging design vocabulary. Our investigation shows us there is similarity even if sometimes there are differences in naming.

One could see the advantage of what we are taking about if we look at Advanced Message Queuing Protocol (AMQP) [37] practice when it standardized message format (known as a wire-level protocol) which is proprietary in JMS [38]. Any tool conforming to this data format can interoperate with any other compliant tool regardless of implementation language. Both JMS and Microsoft's MSMQ [39] comprise alternative candidate platforms for our messaging system PSM. Both have similar capabilities but have differences in performance and integration features plus others. Our messaging PSM is developed from the standard of JMS which is recognized as the best-known standard in the asynchronous messaging world [40]. As we mentioned, a complete ontology of messaging systems needs to be established by a standards body so one could take the advantage of replacing one messaging platform by another. This standard will establish a vendor-neutral protocol by studying different practices of messaging paradigms such publish/ subscribe, point-to-point, request-response, etc. The standard would specify message format, Brokers behaveior scenarios, and others.

\section{Conclusions}

MDA is about mapping PIM instances to PSM instances automatically using a standard mapping language such as QVT as a new trend of developing applications. The MDA standard specification does not show in details how to do the mappings from PIM metamodel (application-space) to PSM metamodel (implementation-space). This situation raises a question: how to develop End to End applications which is the ultimate goal of MDA.

In the view of that question we have proposed E2EDE, a novel software development approach which bridges the mapping gap between PIM (functional specification) and PSM (implementation specification) using the MDA method.

E2EDE approach is based on documenting variability in architecture artifact design on the PSM by utilizing the variability notion in the software product line approach. Our variability analysis has shown taxonomy for variability including mapping variability.

NFRs is proposed to be documented in PIM to make the PIM more informative thereby guiding the mapping process to select from among design alternatives in order to automatically produce a suitable implementation or PSM instance model. In this scenario the mapping process is modeled in a configurable way to drive an architecture that can lead to considerable cost-saving. We have shown that this study has contributed to NFRs knowledge by identifying two kinds of NFRs: Package level and class level. The former have more re-use potential.

E2EDE contributes to the MDA domain by finding concrete mapping methods for generating high quality applications within specific but big enough domains through building explicit links between design decisions and NFRs.

E2EDE implementation models were developed and it was discovered that a profile is good at presentation level but not suitable for implementation level. Generally, we followed Profiles, Packages, and model manipulation approach where metamodels were developed for source, target and relationship program. 
We have investigated the realistic application of E2EDE and found that there different examples of messaging systems without a standard. For use of E2EDE, having a standard PSM would be an advantage. It increases the reuse theme (PIM with NFRs can be like variant feature) and achieves interoperability. The best situation would be gained if PSM is built by standards bodies such as ISO or the OMG.

Finally, throughout this paper we have seen how MDA can fit in with SPL and Design pattern under the reuse umbrella which helps explore the research issues that are arose such as Non-functional requirements when we tackle E2EDE engineering. A case study was presented to show the possibility of success under this approach. A strategic PSM for messaging systems is developed as another potentially valuable product. In addition, the lessons and the realistic application of the approach are investigated.

\section{REFERENCES}

[1] “MDA Guide Version 1.0.1,” 2001. http://www.omg.org/cgi-bin/doc?omg/03-06-01.

[2] K. Pohl, G.Böckle and F. J. van der Linden, "Software Product Line Engineering: Foundations, Principles and Techniques,” Springer, Berlin, 2005, pp. 53-72.

[3] H. Min and S. D. Kim, "A Technique to Represent Product Line Core Assets in MDA/PIM for Automation," Proceedings Rapid Integration of Software Engineering Techniques Second International Workshop (RISE 2005), Minneapolis, Vol. 3943, 2006, pp. 66-80.

[4] M. Matinlassi, "Quality-Driven Software Architecture Model Transformation,” PhD Dissertation, VTT Technical Research Centre of Finland, 2006. www.vtt.fi/inf/pdf/publications/2006/P608.pdf

[5] S. J. Mellor, K. Scott, A. Uhl and D. Weise, "MDA Distilled: Principles of Model-Driven Architecture,” Addison Wesley, New York, 2004.

[6] R. M, Colomb "Metamodelling and Model-Driven Architecture,” In Publishing.

[7] “MOF 2.0 Core Final Adopted Specification,” 2004. http://www.omg.org/cgi-bin/doc?ptc/03-10-04.

[8] “OMG MOF QVT Final Adopted Specification,” 2005. http://www.omg.org/docs/ptc/05-11-01.pdf

[9] S. Jarzabek, "Effective Software Maintenance and Evolution: A Reuse-Based Approach,” Auerbach Publications, Boca Raton, 2007, pp. 68-106. doi:10.1201/9781420013115

[10] D. Ramljak, J. Puksec, D. Huljenic, M. Koncar and D. Simic, "Building Enterprise Information System Using Model Driven Architecture on J2EE Platform," Proceedings IEEE the 7th International Cconference on Telecommunications, Zagreb, June 2003, Vol. 2, pp. 521-526.

[11] D. Muthig and C. Atkinson, "Model-Driven Product Line Architectures," Second International Conference on Soft- ware Product Lines, San Diego, Vol. 2379, August 2002, pp. 79-90.

[12] B. Korherr, "A UML2 Profile for Variability Models and Their Dependency to Business Processes,” Proceedings of IEEE Conference Database and Expert Systems Applications, Regensburg, September 2007, pp. 829-834.

[13] L. Chung "Representing and Using Non-Functional Requirements: A Process-Oriented Approach,” PhD Thesis. University of Toronto, Toronto, 1993.

[14] D. Gross and E. Yu, "From Non-Functional Requirements to Design through Patterns," Requirements Engineering, Vol. 6, No. 1, 2001, pp. 18-36. doi:10.1007/s007660170013

[15] F. Buschmann, K. Henney and D. C. Schmidt, "Pattern Oriented Software Architecture on Patterns and Pattern Languages,” John Wiley \& Sons, England, Vol. 5, 2007, pp. 67-74.

[16] M. Svahnberg, J. Van Gurp and J. Bosch, “A Taxonomy of Variability Realization Techniques,” ACM SoftwarePractice \& Experience, Vol. 35, No. 8, July 2005, pp. 705-754.

[17] R. Elmasri and S. B. Navathe, "Fundamentals of Database Systems," 5th Editon, Addison-Wesley, Reading, 2007, pp. 232-234.

[18] I. Dubielewicz, B. Hnatkowska, Z. Huzar and L. Tuzinkiewicz, "Feasibility Analysis of MDA-Based Database Design,” IEEE International Conference on Dependability of Computer Systems, Washington, May 2006, pp. 19-26. doi:10.1109/DEPCOS-RELCOMEX.2006.26

[19] M. Glinz, “On Non-Functional Requirements,” Proceedings of the 15th IEEE International Requirements Engineering Conference, Delhi, October 2007, pp. 21-26.

[20] Wikipedia, "X Window System (Computer Science)," 2008. http://en.wikipedia.org/wiki/X_window_system.

[21] L. Bass, P. Clements and R. Kazman, "Software Architecture in Practice," 2nd Edition. Addison-Wesley, Massachusetts, 2003, pp. 75-88.

[22] L. Zhu and I. Gorton, "UML Profiles for Design Decisions and Nonfunctional Requirements," IEEE Second Workshop on Sharing and Resuing Architectural Knowledge, Minneapolis, May 2007, pp. 49- 54.

[23] F. J. V. Linden, K. Schmid and E. Rommes, "Software Product Lines in Action: The Best Industrial Practice in Product Line Engineering,” Springer, Berlin Heidelberg, 2007, pp. 43-45.

[24] C. Alexander, S. Ishikawa, M. Silverstein, M. Jacobson, I. Fiksdahl-King and S. Angel, "A Pattern Language,” Oxford University Press, New York, 1977.

[25] Wikipedia, “Design Patterns (Computer Science),” 2008. http://en.wikipedia.org/wiki/Design_pattern_\%28comput er_science $\% 29$

[26] E. Gamma, R. Helm, R. Johnson and J. Vlissides, "Design Patterns: Elements of Reusable Object-Oriented Software,” Addison-Wesley, New York, 1995, pp. 79315.

[27] M. Yacoub, H. Xue and H. Ammar, “Automating the 
Development of Pattern-Oriented Designs for Application Specific Software Systems," Proceedings IEEE the 3rd Symposium on Application-Specific Systems and Software Engineering Technology, Washington DC, March 2000, pp. 163-170.

[28] S. M. Yacoub, "Pattern-Oriented Analysis and Design (POAD): A Methodology for Software Development," PhD Thesis, West Virginia University, Morgantown, December 1999.

[29] S. J. Mellor, K. Scott, A. Uhl and D. Weise, "MDA Distilled: Principles of Model-Driven Architecture,” Addison Wesley, New York, 2004.

[30] “OMG MOF XMI Final Adopted Specification,” July 2010.

http://www.omg.org/technology/documents/formal/xmi.ht $\mathrm{m}$.

[31] P. Konemann, “The Gap between Design Decisions and Model Transformations," September 2009. http://www2.imm.dtu.dk/.../the_gap_between_design_dec isions_and_model_transformations.pdf

[32] D. Steinberg, F. Budinsky, M. Paternostro and E. Merks, "EMF: Eclipse Modeling Framework," 2nd Edition, Addison-Wesley Professional, Singapore, December 26
2008.

[33] “IKV++ technologies ag.MediniQVT,” 2007. http://projects.ikv.de/qvt/

[34] “W3C OWL Web Ontology Language,” August 2010. http://www.w3.org/TR/owl-ref/

[35] "OMG (2007b) OMG Unified Modeling Language (OMG UML), Superstructure, V2.1.2,” OMG Document Number: Formal/2007-11-02.

[36] W. R. Sebesta "Concepts of Programming Languages," 5th Edition, Addison Wesley, Boston, 2005.

[37] “Microsoft Messaging Queue,” August 2010. http://www.microsoft.com/windowsserver2008/en/us/ technologies.aspx.

[38] “Advanced Message Queuing Protocol (AMQP),” 2010. http://www.amqp.org/confluence/display/AMQP/Advanc ed+Message+Queuing+Protocol

[39] “Java Messaging System Standard,” 2010. http://java.sun.com/products/jms/

[40] S. Vinoski, “Advanced Message Queuing Protocol,” IEEE Internet Computing, Vol. 10, No. 6, 2006, pp. 87-89. doi:10.1109/MIC.2006.116 